862 Verhandlungen der Berliner dermatologischen Gesellschaft.

Herr O. Rosentlal dankt für die interessanten Krankenvorstellungen und Ausführungen, besonder's aler Herrn Buschke für die Veranstaltung und Leitung dieser Sitzung im Namen der Berliner dermatologischen Gesellschaft.

I m merwabr (Berlin).

\title{
Verhandlungen der Société française de Dermatologie et de Syphiligraphie.
}

Sitzung vom 8. Januar 1919.

Ansprache des Präsidenten Renault, worin er seine Befriedigung über den Sieg ausdrückt, der verstorbenen Mitglieder gedenkt, namentlich Fourniers, Jacquets und $G a d u c h$ ers und zu neuer wissenschaftlicher Arbeit aufmuntert, die der Kriegsanstrengung ebenbürtig sein soll.

Leredde. Die Behandlung der Syphilis während der Kriegszeit. Methoden und Resultate. Leredde glaubt, daß die Luetiker in der Armee nur unzureichend behandelt worden seien und übt Kritik an dem Rundschreiben des Unterstaatssekretärs. Er schätzt die Neuinfektionen in der Armee auf Hunderttausende. Er glaubt. daß $3 / 4$ derselben nach dem Kriege ungeheilt blieben und die Krankheit in der Heimat weiter verschleppen. Gleich bei Ausbruch des Krieges hätte viel energischer gegen die Krankheit vorgegangen und nach den Erfahrungen der Vorkriegszeit die Sterilisation angestrebt werden sollen.

Diskussion. Simon und Milian halten die von Lere d de angegebenen Zahlen von Neuinfektion für kolossal überschätzt. Milian schätzt die Zahl der jährlichen Neuinfektionen in der Armee auf ungefähr 5000 und nimmt die Behandlungsmethode in der Armee gegenüber den Angriffen von Leredde in Schutz. Er wird dabei unterstützt von Gougerot, der die Behandlung des Militärs während des Krieges für besser bält als die der Zivilpersonen. Auf die Frase von Thibierge, wie lange ein Syphilitiker im Spital behalten werden sollte, antwortet Leredde: 2-3 Monate d. h. bis die Wa.-R. negativ geworden ist.

Gougerot. Bazilläre Dermoepidermatitiden des Krieges (strepto- und staphylogen). Gougerot hebt die große Bedeutung der Dermoepidermatitis während des Krieges hervor, die, nicht erkannt, zu monatelanger Spitalsbehandlung führe und den Mann dienstuntauglich mache, während sie bei richtiger Therapie in 2-6 Wochen zur Heilung gebracht werden könne. Die Krankheit stellt eine Infektion der Kutis und insbesondere der Epidermis dar, befällt gewöhnlich große Hautflächen und ist bedingt durch Strepto- und Staphylokokkeninfektionen oder beide zusammen. Sie ist hauptsächlich um verletzte Hautstellen lokalisiert. Er unterscheidet eiternde, nässend-erosive, ekzematoide, trockene, schuppeude und polymorphe Formen mit einer großen ZahI von Uuterabteilungen. 
Verhandl. der Soc. tranç. de Dermatologie et de Syphiligr. 863

Jamin. Über 29 Fälle von Arsenexanthem. Jamin beobachtete in einer dermatologisch-venerologischen Abteilng in Tunis 29 Fälle von Arsenexanthem bei eingeborenen Rekruten, die, um sich der Dienstpflicht zu entziehen, ein Gemisch von Thapsia und Honig mit Beimengung von Arsentrisulfat eingenommen hatten. Fine cinzige Einnahme dieser Mischung hatte genügt, um folgende Intoxikationserscheinungen hervorzurufen: Am 2. Tage nach der Einnabme zeigten sich mit oder ohne Allgemeinerscheinungen linsengroße, runde, rote Flecke an der Streckseite der Extremitäten, die am anderen Tage zusammenflossen und sich allmählich äber den ganzen Körper ausbreiteten, der dann wie bei einer akuten Erythrodermie aussah. Im allgemeinen konnte der Vortragende 2 Formen unterscheiden: 1. eine leichte Form, bei der Allgemeinsymptome fehlten und bei der auch die Mundschleimhant verschont blieb. Nach 3 Tagen erfolgt eine kleienförmige Abschilferung wie nach Masern. Kein Pruritus. 2. Eine schwere Form, bei der es zu allgemeiner Mattigkeit, Kopfschmerzen, Diarrhoen, Appetitlosigkeit, häufig Epistaxis und Fieber bis 40 kommt. Das Exanthem, anfänglich morbilliform, wird bald skarlatiniform. Dabei können quaddelartige Papeln, Erythema exsudativum multiforme und erysipelartige Plaques auftreten, die aber alle nur vorübergehenden Charakter haben, um einer gleichmäßigen Rötung zu weichen. Auch Blasen vom Charakter der Brandwunden sollen dabei vorkommen. Bei einigen Fällen bestand Jucken. Auch die Mundschleimhaut wird betroffen in Form von Blasen wie auf der Haut. Der Temperaturabfall erfolgt lytisch, ebenso geht der Puls zurück und kann bis unter 50 pro Minute fallen. Das Allgemeinbefinden liegt noch lange Zeit wie bei Typhus darnieder. Im Urin häufig Albumen. Die Abschuppung erfolgt lamellenförmig, oft in Form von großen Fetzen. Nach der Abschuppung bleibt noch eine Monate lang dauernde Pigmentierung zurück.

Jamin. Fall von Erythrodemia exfoliativa nach der Einnahme eines "cachet Faivre" (Oxichinothein). Es wird eine Moulage des Falles von Darier demonstriert.

Milian. Épidermocanaliculitis gonorrhoica an der Penis raphe. Milian sah bei einem Soldaten einen ungefähr zentimeterbreiten Strang an der Penisraphe, der entzündlich gerötet und vorspringend war und aus verschiedenen Fisteln Eiter entleerte. Der Eiter enthielt Gonokokken. Die Affektion soll sich bei einer Urethralgonorrhoe entwickelt haben und schon 6 Monate bestehen. Der Strang wurde exzidiert und histologisch untersucht. Es zeigte sich, daß nicht, wie klinisch vermutet wurde, eine Lymphangitis vorhanden war, sondern daß es sich um einen mit Pflasterepithel ausgekleideten Kanal handelte, der auf einen mangelhaften Verschluß des urogenitalen Kanals zurückzuführen und gonorrhoisch infiziert war.

Chadzynski. Welches ist die wirkliche Bedeutung der Wassermannschen Reaktion für die Diagnose und Behandlung der Syphilis? Der Autor kommt angesichts der vielen mög- 
lichen Irrtümer, die teils in den Laboratorien vorkommen, teils in dem zufälligen Zustand des Patienten gelegen sein können und da die Reaktion nicht spezifisch sei und die Patienten in falsche Sicherheit wiege, zum Schlusse, daß die Wa.-R. mehr gefährlich als uätzlich sei und daß man sich deshalb weder bei der Diagnose noch bei der Therapie von ihr leiten lassen solle.

Diskıssion. In der Diskussion werden die offenbar noch unter dem Kriegseinfiuß leidenden, etwas leichtfertigen Ausführungen des polnischen Vertreters von Milian, Sre und leredde einer berechtigten Kritik unterworfen und die Bedeutung der Wa.-R. ins richtige Licht gestellt, während sie von Ravaut als nicht absolut beweiskräftig für die Diagnose der Lues bezeichnet wird. So könne sie bei Lepra, Skarlatina, bei Angina $V$ incent $i$ und bei Tuberkuliden positiv sein, während sie hie und da auch bei manitesten Erscheinungen negativ ausfalle.

\section{Sitzung vom 13. März 1919.}

Milian, G. Durch Hg geheilte Choreasyphilitica. Fall von Chorea bei einem 2ljährigen Mädchen, das Zeichen von hereditärer Lues und eine positive $\mathrm{Wa}-\mathrm{R}$. aufwies. Auf $1^{1 / 2} \mathrm{eg} \mathrm{Hg}$ cyanatum intravenös starke Steigerung der choreatischen Bewegungen während 48 Stunden, diese Verschlimmerung wird von Milian auf die Herxheimersche Reaktion zurückgeführt. Unter weiterer $\mathrm{Hg}$-Behandlung erfolgte in kurzer Zeit vollständige Heilung. Dieser Fall bestärkt den Verf. in seiner Ansicht von der syphilitischen Natur der Chlorea.

Diskussion. Nach Quayrat ist Milian zu exklusiv mit seiner Annahme der syphilitischen. Ätiologie dor Chorea. Man müsse neben der Lnes auch eine gut definierte infektiöse Chorea annehmen, die rheumatischen Ursprungs sei. Danelen. gebe es eine hysterische Chorea. Anch Balaer glanbt, dali es neb'n einer rheumatischen eine syphilitische Chorea gebe. Mirian gibt $\mathrm{zu}$, dab es neben der Lues noch andere Infektionen gebe, die Chorea erzeugen können.

Blum, P. Fall von Parapsoriasis gutata.

Fall von Parapsoriasis in Form von hanfsamen- bis linsergroßen, leicht schuppenden, schwach roten Flecken, die generalisiert über den Körper verbreitet sind bei einem 19jährigen Manne. Patient weist Zeichen einer tuberkulösen Lungenaffektion auf. Der histologische Befund sprach für eine Paräpsoriasis.

Fernet und Kolopp. Fall von Xanthoerythrodermia perstans.

Verf. stellen einen Fall von Xantho-Erythrodermia perstans bei einem 36jährigen Manne vor. Die von $\mathrm{Crocker}$ beschriebene Affektion zeigt sich in Form von rundlichen bis ovalen, kaum über die Haut erhabenen schwach roten bis ockerg llben, nagel- bis 2 frankstückgroßen Flecken, die an der Stirn, am Rumpf und den Beinen lokalisiert sind. Bei der Palpation zeigt sich eine leichte Infiltration. Schuppung sehr unbedeutend. W.-R. negativ.

Brocq, in dessen Abteilung der Fall zur Beobachtung kam, hält die Affektion für nahe verwandt mit der Parapsoriasis. Die stärkere Ocker- 
farbe, die größere Widerstandskraft gegenüber dem "grattage métodique * und die weniger ausgesprochene Schuppenbildung lasse sie aber von der Parapsoriasis unterscheiden.

Fernet und Scheikievitch demonstrieren einen Fall von Erythrodermia generalisata mit Drüsenschwellung bei einem 61jährigen Manne. Neben der Rötung bestand Infiltration und Schuppenbildung, sowie stellenweise (Gelenksfalten) leichtes Nässen und zeitweiser intensiver Pruritus. Trotz Röntgen-Bestrahlnng zunehmende Drüsenschwellung und Hypertrophie der Tonsillen. An den Handtellern und Fußsohlen Keratodermie. Nägel deformiert. Im Blute eine leichte Leukozytose. Der histologische Befund von Darier lautet auf Leukämieverdacht.

Jeanselme und Touraine demonstrieren einen Fall von zosterähnlich anf der Rückseite des linken Arms und der linken Thoraxseite lokalisierter Psoriasis. Sie machten dabei die Lumbalpunktion und fanden aber nur unbedeutende Abweichung der Lumbalflüssigkeit. Trotzdem wollen sie die Beteiligung des Nervensystems bei diesem Fall nicht ganz ausschließen.

Bloch und Lévy demonstrieren einen 25jährigen Soldaten mit einem $\mathrm{Lupus}$ am linken untern Augenlid und einer Infiltration an der Vorder- und Seitenfläche links am Hals, die mehrmals punktiert wurde und bei der jeweilen Eiter entleert werden konnte. Es handelt sich um ein großes Infiltrat, das aus nußgroßen Knoten zusammengesetzt ist, die im Unterbautzellgewebe liegen. Die Drüsen sind intakt. Histologisch handelt es sich um abgekapselte Herde von Lymphozyten und Riesenzellen ohne Tuberkelbildung. Tuberkelbazillen konnten nicht gefunden werden. An einigen Stellen peripheriewärts konnten Tuberkelbildungen konstatiert werden. Sarkoid.

Diskussion. Darier hâlt die Affektion am Hals für ein subkutanes

Gougerot und Bonn in demonstrieren einen Fall von Granuloma annulare an den Händen bei einer 36jährigen Frau ohne tuberkulöse Antezedentien. Patientin zeigt mehrere Herde an den Fingern und einen Herd an der Ulnarseite der rechten Hand. Die Entwicklung ging nicht exzentrisch vor sich, sondern soll mehr serpentinenartig verlaufen sein Histologisch handelt es sich nicht um ein Granulom, sondern um einen tuberkuloiden Bau der Läsionen. $O b$ die Affektion den Tuberkaliden zuzurechnen ist, wagen die Autoren nicht zu entscheiden.

Gougerot. Fall von netzförmig angeordnetem $\mathrm{Pemphigus}$ an der Vorderseite der Oberschenkel, der Frühling und Herbst rezidiviert bei einer 40jährigen Frau. Die Blasen haben unregelmäßige Formen, sind länglich, sternförmig usw. und konfluieren schließlich zu gewaltigen flasken Säcken. Die Affektion entwickelt sich gewöhnlich in 3-5 Tagen, verläuft unter leichtem Jucken ohne Alteration des Allgemeinbefindens. Nach Abheilung der Blasen bleibt eine lichenoide papulo-squamöse Haut an Stelle der affizierten Hautpartien zurück. Die Epidermis ist leicht 
verdickt, etwas squamös, schwach rosarot bis bräunlich violett verfärbt, etwas pigmentiert, wie bei Lichen keratosus.

Broussolle, J. demonstriert 2 Kabylen, die in einer Teerdestillationsfabrik beschäftigt waren und an Akne im Gesicht and zum Teil an den Beinen und Vorderarmen litten. Die zu leistende Arbeit bestand in der Bedienung der Naphthalinpresse, wobei sie sich oft auf die Presse legten. Bei dem Verdampfen gingen öldämpfe in die Luft über, die besonders beiliend gewesen sein sollen, und mit denen das Gesicht, die nackten Streckseiten der Vorderasme und die Knie der Arbeiter in Berührung kamen. Broussolle macht die Öldämpfe des Naphthalins für die Entstehung der Akne verantwortlich, wobei die Seborrhoe als begünstigendes Moment wirkt. Die Akne bestand hauptsächlich aus Komedonen und zum geringen Teile aus Akneknötchen. Pusteln fehlen ganz. Eine gute Körperpflege, bestehend in reichlichen Seifenwaschungen, soll das beste Prophylaktikum sein.

Milian demonstriert einen Mann, der wegen einer syphi!itischen Hemiplegie 12 Injektionen von je $\mathrm{l}$ cg $\mathrm{Hg}$ cyanat. intravenös bekommen hatte und zwar 3 Injektionen pro Woche. Trotz dieser Behandlung entwickelte sich auf der rechten Kopfseite eine gewaltige syphilitische Ulzeration, an der Stelle, wo Pat. einige Monate früher ein Trauma erlitten hatte. Auf tägliche Injektionen von $\mathbf{H g}$ cyanat. in toto 20 heilte die Ulzeration. Milian warnt vor zu schwachen, zu kurzen und unregelmäBig fortgesetzten Behandlungen, da sie das Erscheinen von luetischen Erscheinungen provozieren können.

Diskussion. Milian wird unterstützt von Leredde, der in solchen Erscheinungen eine Reaktivation - sieht, während Darier meint, daß die ungenügende Behandlung einfach die weitere Entwicklung einer luetischen Läsion nicht verhindert habe.

Simon und Gastinel haben an Hand von 90 Primäraffekten vor, ${ }^{\prime}$ ährend und nach der Behandlung vergleichsweise die W a s e r m a n $n$ sche und $\mathrm{Hechtsche} \mathrm{Blutreaktion} \mathrm{geprüft} \mathrm{and} \mathrm{gefunden,} \mathrm{daB} \mathrm{die} \mathrm{Hecht}$ sche Reaktion früher, regelmäßjger und dauernder zu konstatieren ist als der Wassermann. Trotzdem glauben sie nicht, daB die W.-R. durch $\mathrm{Hecht}$ ersetzt werden könne, da man sonst leicht zu falschen Resultaten gelangen könne, teils wegen Überschuß, teils Fehlens des natürlichen Komplements oder Hämolysins der Sera.

Simon und Couratte-Arnaude empfehlen zur Epilation das Calcium sulfuricum besonders bei Follikulitiden, Pediculi pubis, Ekzem der behaarten Körperteile, Impetigo. Die Epilation gehe ohne Hautreizung vor sich und das Epilatorium entfalte zugleich eine desinfizierende Wirkung. Die Nachteile desselben sind die geringe Haltbarkeit und der stechende Geruch des Mittels, weshalb es sich hauptsächlich für die Spitalbehandlung eigne.

Du Castel teilt einige Fälle von Basedow scher Erkrankung mit, bei denen Dermatosen auftraten und zwar beobachtete er Alopecia areata, Ekzeme und Pyodermien. Er glaubt, daß diese Affektionen wegen 
des zeitlichen Zusammentreffens in einer gewissen Beziehung zum Basedow stehen könnten.

Du Castel empfiehlt zur Behandlung der Draconculose (Filiariaerkrankung) Einreibungen mit Unguentum cinereum. Einerseits werden dadurch die Infiltrate resorbiert, andererseits die Würmer getôtet. Du Castel teilt die Krankengeschichte eines von ibm beobachteten Falles kurz mit.

Diskussion. Jeanselme und Goubeau wollen Fälle von Filiaria mittels intravenöser Novarsenobenzolinjektionen geheilt haben.

Touraine hat bei 10 Fällen von Vitiligo die Lumbalpunktion gemacht und folgendes konstatiert: In 8 Fällen war die Reaktion abnormal und zwar war bei 5 Patienten die W.-R. positiv, es bestand Hyperalbuminurie und Lymphozytose. Bei 3 andern Fällen fehlte die eine oder andere Reaktion und nur 2 Fälle verhielten sich normal auch nach der Reaktivation. Die Veränderungen zeigten sich um so deutlicher, je jünger die Fälle waren, während schon lange bestehende Fälle 12 und 27 Jahre - eine normale Reaktion aufwiesen.

Milian empfiehlt bei Lues verdächtigen Patienten, die eine positive W.-R., aber eine negative Anamnese und einen vollkommen negativen Status aufweisen, die Diagnose ex juvantibus zu versuchen. Er hat in einem solchen Falle ein deutliches Schwächerwerden der Reaktion nach der Hg-Kur beobachtet. Zum Vergleiche empfiehlt er auch andere Affektionen mit positiver W.-R. wie die Lepra zu prüfen.

Goubeau demonstriert einen Senegalesen mit pigmentierten Plaques a u der $Z$ unge und zwar an Stelle von sog. „Plaques fauchées". Bei einem andern Falle sollen diese Pigmentierungen auf die spezifische Behandlung hin nicht verschwunden sein.

Diskussion. Darier schlägt vor, diese Affektion nicht Pigmentsyphilis zu nennen, sondern Syphilides nigricantes, welcher Ansicht sich auch $\mathrm{Bal}$ \% e $\mathrm{r}$ anschließt. Milian hat einen analogen Fall in Beobachtung.

Fernet. 35jähriger Mann erkrankt an Lues und bekommt innerhalb eines Jahres ungefähr $24 g$ Novarsenobenzol und 5 c $g \mathrm{Hg}$ cyanat. Trotz dieser intensiven Arsenmedikation bekommt Patient wieder erosive Läsionen am Skrotum, die auf Jod und $\mathrm{Hg}$ rasch abheilen. Fernet glaubt, daß in diesem Falle eine spezielle As-Resistenz bestanden oder daß eine allmähliche Angewöhnung des Organismus stattgefunden habe.

Sitzung rom 10. April 1919.

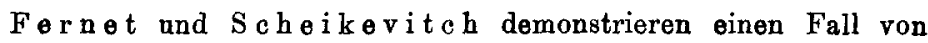
generalisierter Erythrodermie mit Hyperepidermotrophie sowie Läsionen vom Charakter der Pityriasis rubra pilaris an den Fingerrücken, den Handgelenksrücken und am Hals. Es bestehen anch Veränderungen von der Form der Sklerodaktylie und sklerodermatischer Atrophie am übrigen Körper. Die Veränderungen sind nicht kongenital, sondern haben sich erst in den letzten 5 Jahren bei einem 51jährigen Manne 
entwickelt. Auf Schilddrüsenpräparate soll jeweilen eine vorübergehende Besserung eingetreten sein. Diagnostisch wird der Fall am ebesten für cine Übergangsform von Pityriasis rubra pilaris und Erythrodermia ichthyosiformis mit Hyperepidermotrophie, Dermoatrophie und Sklerodaktylie gebalten.

Fernet stellt einen Fall zur Diagnose vor. Es handelt sich

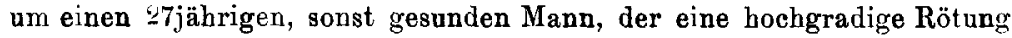
der gesamten Körperhaut, mit Ausnahme des Kopfes, Halses und der Beugefalten aufweist. Auch die Handteller und FuBsohlen sind kaum betroffen. Allgemeinbefinden sehr gut. Die Rötung ist fast scharlachartig. Dazu besteht eine teils kleienförmige, teils lamellöse Schuppenbildung. Mikroskopisch Dilatation der Blutgefäße und Infiltration mit Lymphozyten und fixen Bindegewebszellen. Im Blute eine leichte Eosinophilie. Subjektiv kein Jucken. Es werden differentialdiagnostisch eleminiert die Dermatitis exfoliativa acuta, subacuta, die Pityriasis rubra $\mathrm{Hebrae}$, die Erythrodermia praemycotica fungoides und die Leukämide.

'I'hibierge und Mercier demonstrieren eine 24jährige Frau, die nach Novarsenobenzolinjektionen rote Flecke bekam, die allmählich einen bräunlichen Farbenton anehmen, ähnlich den Antipyrinexanthemen.

Hudelo und Chabanier. Fall von starker Lymphombildung am Hals und im Mediastinum mit pruriginösen Läsionen auf der Haut. Im Blute eine leichte Leukozytose. Der Fall wird von den Autoren als "Prurigo lymphadénique" bezeichnet, in Deutschland unter dem Namen Lymphogranulomatose bekannt.

Fernet und Scheikevitch demonstrieren einen Fall von generalisierter Vakzine bei einem Stallknecht.

Jeanselme und Touraine. Fall von Urticaria pigmentosa mit starker Hypertrophie der Milz und leichter Vergrößerung der Leber bei einem 41jährigen Manne. Die Affektion soll im Alter von 15 Jahren begonnen haben und schubweise aufgetreten sein. Im Blute positive Wa.-R., im Lumbalsekret ist sie negativ. Das Blut zeigt eine leichte Anämie und Leukopenie. Auf Röntgen-Bestrahlungen der Milz tritt eine bedeutende Verkleinerung derselben sowie der Leber ein. Das Blutbild wird besser und die Schübe von Urticaria pigmentosa verschwanden. Die Dermatose ist in Rückbildung.

Diskussion. Gestützt auf den positiven Wa. wünscht Leredde eine genauere Untersuchung auf Lues und antiluetische Behandlung. Pellie: vermißt die Biopsie. Jeanselme läbt klinisch keine $Z$ weifel an der Diagnose zu. $\mathrm{Ob}$ der Urticaria pigmentosa ätiologisch eine Lues zugrunde liege, könne nicht entschieden werden. Simon hat einen Fall von U. p. gesehen, bei dem die Wa.-R. negativ war.

L a c a p̀̀ re weist auf die Differénzen der Toxizität der einzelnen Novarsenobenzolampullen hin. Es ist ihm aufgefallen, daß manchmal bei einer Serie von Injektionen der Dosis 0.45 starke toxische Wirkungen 
aufgetreten sind, während mit stärkeren Dosen einer anderen Serie die Nebenerscheinungen ganz unterblieben. Er empfieblt eventuell gleichzeitig mit dem Neosalvarsan subkutan Adrenalin zu injizieren.

Diskussion. Pautrier bestätigt die Ausführungen von $\mathbf{L}$ a c a père. Einzelne Serien erwiesen sich als defekt. Anch Lévy-Franckel hat üble Zufälle bei gewissen Serien von Novarsenobe und sogar 2 Todesfälle gesehen. Es sei nötig, sich die Nummern dts ampullen zu merken, die zu Yebenerscheinungen führen. Pinard macht auf den verschiedenen Gehalt an As der französischen Präparate und des deutschen Präparates aufmerksam. Die französischen Präparate sollen 21.42 und $27.44^{\circ}, 0 \mathrm{As}$, das deutsche $2.74 \%$ enthalten. Die ideale Formel sollte nach Cous in $32 \cdot 18 \%$ As Gehalt haben. Emery hat ebenfalls mit gewissen Serien unangenehme Zufälle erlebt. Daneben empfiehlt er zur Vermeidung der Krisen sehr langsames Einspritzen der Lösungen und Verwendung von vorgängigen kleinen Dosen. Leredde behauptet, daß ein gut zubereitetes Novarsenobenzol nicht toxisch sei. Die Herxheimersche Reaktion sei die gewöhnliche Ursache des Todes. Die inneren Organe werden in der Regel zu wenig untersucht und andererseits seien die Dosen vielfach zu hoch, so im Falle von Bory. Bory wehrt sich; bei seinem Falle sei der Tod nach der 4. Injektion - Dosis 0.75 - aufgetreten, nachdem 0.3, 0.15 und 0.6 gut vertragen worden seien.

Gastinel und Pellier. Fall von Favus am Skrotum bei einem 15jährigen Knaben.

Du Castel hat bei einem Bataillon von annamitischen Jägern eine Epidemie von Achromie beobachtet, die speziell auf das Gesicht, den Hals und die oberen Thoraxpartien beschränkt war und sich in Form von kleinen und z. T. größeren unregelmäßigen Flecken zeigte. $\mathrm{D} \mathrm{u} \mathrm{Cas} \mathrm{tel}$ nimmt eine parasitäre Ätiologie an. 3 Fälle werden etwas eingehender besprochen.

\section{Sitzung rom 3. Mai 1919.}

L eredde sucht seine in der früheren Sitzung gemachte Angabe über die Zunahme der Syphilis während des Krieges um mehrere Hunderttausende durch neue Argumente zu begründen und hält sie aufrecht.

Diskussion. Gaston verlangt eine genaue Statistik, da manchmal derselbe Fall zweimal gezählt worden sei.

Ba]zer. Fall von kongenitaler Dermoidzyste auf der Unterseite des Penisschaftes, die gon or $\mathrm{rhoisch}$ infiziert und durch eine zweimalige Exzision beseitigt wurde.

L e redde bespricht die Bedingungen, unter denen die meisten Todesfälle bei Salvarsanbehandlung eingetreten sind. Neben Fehlern der Technik - zu große Dosen im Beginn der Behandlung - spielt die Herxheimersche Reaktion nach dem Autor die Hauptrolle. Die Patienten müssen in Bezug auf das Nervensystem und die inneren Organe vor Beginn der Kur sehr genau untersucht werden, Es müsse die Toleranz der Kranken stets durch sehr kleine Dosen eruiert werden. Nachher könne dann allmählich zu sehr hohen Dosen, bis 0.9 , ja 1.2, übergegangen werden. 
Lacapère macht Mitteilung von 5 Todesfällen, die bei der Behandlung mit Novarsenobenzol aufgetreten sind. Beim ersten Fall handelte es sich um eine Blutung im Bereiche der Protuberantia. Der Tod ist nach einer Injektion von 0.45 Novarsenobenzol aufgetreten, nachdem 0.15 und 0.3 gut vertragen worden waren. Beim 2. Patienten ebenfalls nach 0.45 Novarsenobenzol Tod im Koma. Keine Autopsie. Verf. bält es für wahrscheinlich, daß ein toxisches As-Präparat vorgelegen habe. Beim 3. Fall trat der Tod 5 Tage nach einer einmaligen Injektion von $0 \cdot 1$ Novarsenobenzol auf. Beim 4. nach einer Injektion von $0 \cdot 1$ Galyl. Beim 5. Fall handelte es sich um eine Malaria mit erschlafftem Herzmuskel. Der Tod trat nach 0.3 Novarsenobenzol im Kollaps auf.

Diskussion. Leredde glaubt, daß die Herxheimersche Reaktion bei den Todesfällen die Hauptrolle spiele. Er führt einen Fall an, der ihm in diesem Sinne zu sprechen scheint. Sabouraud glaubt sicher zu sein, dab es toxische Serien von Novarsenobenzol gebe. Fr hat bei der gleichen Nummer einer Serie bei 45 Patienten Nebenerscheinungen gesehen. Gongerot glaubt, daß zur größten Sicherheit kleine Mengen eines frischen Lotes in den größeren Spitalsabteilungen geprüft werden sollten, bevor das Präparat in den Handel gegeben werde. Milian will keine toxischen Serien gesehen haben. Er meint, daß manchmal kleine Risse in den Ampullen Schuld an den üblen Zufällen sein könnten. Thibierge glaubt, daß es nur vereinzelte schlechte Serien gegeben habe. Das Präparat als solches dürfe deshalb nicht als schlecht bezeichnet werden. Emery glaubt nicht, daß im allgemeinen die defekten Präparate schwere Erscheinungen machen. Hingegen nimmt er an, da $\beta$ es Serien gebe, die Ikterus machen können. Milian widerspricht der ikterogenen Theorie des Novarsenobenzols von Emery. Nach ihm ist der Ikterus ein hepatisches syphilitisches Rezidiv. Lévy-Bing hat keine Nebenerscheinungen in Serien gesehen, höchstens vielleicht 5 benigne Fälle von Spätikterus.

Queyrat hat vergleichende Untersuchungen angestellt über die Wirkung verschiedener As-Präparate, denen er bei der Behandlung der Lues I und II bei weitem den Vorzug gibt. Geprüft hat er das 606, Arsenobenzol - das 914 oder Novarsenobenzol und das 102 oder Galyl. Queyrat kommt zum Schlusse, daB das Arsenobenzol resp. Altsalvarsan bei weitem das wirksamste Präparat darstelle. Zugleich soll es am wenigsten gefährlich sein. Mit dem Neosalvarsan will er weniger häufig eine negative Reaktion erzielt haben und letztere war nicht so andauernd zu konstatieren. Die Rezidive sollen häufiger aufgetreten sein. $\mathrm{Er}$ empfiehlt, das 914 aufzugeben. Um unangenehme $Z$ wischenfälle mit dem Arsenobenzol zo vermeiden, empfiehlt er folgende Vorsichtsmaßregeln: Das von der Apotheke bezogene destillierte Wasser mub nochmals mittels єines Destillators aus Glas nachdestilliert werden. Die Kupfergefäße sind zu vermeiden, da das Wasser sonst eine saure Reaktion bokommo. Dem destillierten Wasser sind $7 \% \mathrm{NaCl}$ zuzufügen. Die Lösung des Novarsenobenzols hat im Verhältnis von 1:50 zu geschehen. Bei der Alkalisierung muB eine $15 \%$ ige Sodalösung verwendet werden nnd zwar muB noch ein Drittel der Tropfenzahl zugesetzt werden, die zur Klärung des 
Niederschlages nötig waren, sonst bekomme man keine beständigen Lösungen. Es müsse ein Disodo-Arsenobenzol hergestellt werden. Bei den Nebenerscheinungen glaubt $Q$ ueyrat, daB es sich in vielen Fällen um eine Nebenniereninsuffzienz handle. Dafür scheinen ihm die Hypotension der Arterien, der sog. weibe Nebennierenstreifen, das Verhalten der Lumbalflüssigkeit und der gute Erfolg der Adrenalinbehandlung zu sprechen. Was das Luargol betrifft, so soll es in der Wirkung betreffend das Negativwerden der W.-R. ungefähr dem Novarsenobenzol entsprechen, aber weniger toxisch sein. Es soll aber eine sehr gute Wirkung bei der Syphilis des Nervensystems haben und dort hauptsächlich verwendet werden.

Diskussion. Lafay erklärt die chemischen Urnwandlungen des Arsenobenzols bei der Alkalinisierung resp. Lösung des Präparates. Leredde meint, daß $Q$ u e y at mit seiner Ansicht in Bezug auf die Überlegenheit des Altsalvarsans über das Neosalvarsan vielleicht Recht habe, ohne daß er indessen geneigt ist, so große Differenzen anzunehmen. Was L. energisch fordert, das ist die LumbaIpunktion nach dem Negativwerden der Blutreaktion. Emery teilt die Meinung des Vortragenden und gibt dem 606 ebenfalls den Vorzug.

Gougerot, Clara und Bonnin. Fall von atypischer Psoriasis an den Händen, Vorderarmeli, Knien und Ellbogen, sowie im Gesicht. Die Affektion gleicht klinisch einer Psoriasis guttata. weicht aber durch Besonderbeiten rom gewöhnlichen Bilde der Psoriasis ab. Die Effloreszenzen sind mehr papulös, die Schuppen mehr staubförmig. Das Häutchen fehit und statt der punktförmigen tritt eine mehr diffuse Blutung auf. Auch das histologische Bild weist Besonderheiten auf, so daß die Autoren den Fall vorlänfig nicht genau klassifizieren können.

Gougerot sucht an Hand einiger Beobachtungen den Nachweis zu erbringen, daß die sog. "fausse teigne amiantacée d'Alibert" oder das seborrhoische Ekzem des behaarten Kopfes bakterieller Natur ist und zwar vermutet er in erster Linie Streptokokken eventuell in Verbindung mit Staphylokokken oder einer weiteren Bakterienart. Der Übergang von Impetigo, Intertrigo retroauricularis usw. ins seborrhoische Ekzem scheint ihm für seine Theorie zu sprechen.

Gougerot weist auf den Polymorphismus der strepto- und staphylogenen erythematösen squamösen Dermoepidermitiden hin. Die geläufigsten Formen seien: 1. Die Pityriasis simplex des Gesichtes; nach Sa bo u ra nd streptogener Natur. 2. Die erythematös-squamöse Form, die aussehe, wie wenn die Haut mit Kolloidum überzogen wäre. 3. Die erythematös-squamöse Form, ähnlich einem trockenen Ekzem. 4. Die asbestähnliche Form - teigne amiantacée. 5. Die psoriasisähnliche Form. 6. Die Form "en carapace" mit 1-2 mm dicken Krusten. 7. Die pergamentartige Form.

Diskussion. Gastou macht darauf aufmerksam, daB diese Dermoepidermitiden häufig künstlich unterhalten worden seien, ohne daß eine Fistel vorhanden war. 
Cjément Simon demonstriert einen Dilatator praeputii, dann eine Nadel zur Blutentnahme für die Wa.-R., die analog der strauBschen Nadel ist.

\section{Sitzung vom 12. Juni 1919.}

Fernet und Scheikeviteh demonstrieren einen Fall von Lichen ruber planus und acuminatus, der auch Läsionen an der Mundschleimhaut und herdförmigen Haarausfall darbot. Die Affektion begann mit Ulzerationen an der linken Ferse und an der Zunge, die von den Autoren als traumatisch aufgefaßt wurden. Da zunächst die Frage einer luetischen Infektion in Betracht gezogen wurde, leiteten sie eine antiluetische Behandlung mit Novarsenobenzol und Kalomel ein, die aber ein negatives Resultat ergab.

Fernet und Scheikevitch. Fall von Darierscher Psoro spermosis bei einem 60jährigen Manne, der mit Bläschenbildung am Rumpf begann, vom Charakter eines akuten Ekzems oder einer Impetigo, ziemlich stark juckend. Erst ällmählich bildeten sich festhaftende, kleine, braune, schwarze Krusten, die zum Teil in kleine follikuläre Knötchen eingelassen waren. Der von $\mathrm{D}$ a rier verifizierte histologische Befund war typisch. Therapeutisch gab nur die Röntgenbehandlung einen Erfolg.

Hudelo und Barthélemy demonstrieren eine 46jährige Munitionsarbeiterin, die Granaten zu zählen und zu verpacken hatte. Sie kam dabei mit dem die Granaten umgebenden öl in Berührung. Es entwickelte sich bei ihr eine starke Pigmentierung an den unbedeckten Körperteilen, besonders im Gesicht, am Hals und den oberen Brustpartien. Die Pigmentierung war an diesen Partien gleichmäßig und bronzeartig, während an den Handrücken und Streckseiten der Vorderarme mehr eine punktförmige Pigmentierung bestand. An den letzteren Stellen bestand zudem eine follikuläre Hyperkeratose. Die Autoren führen die Affektion auf die im Öl vorhandenen pulverförmigen Stahlteile zurück und glauben, daß es sich um eine Art Tätowierung handle. Nacb Aussetzen der Arbeit trat denn auch bald Besserung auf.

Burnier. Fall von Mastitis syphilitica diffasa bei einer 42jährigen Ehefrau. Die Affektion trat 8 Jahre nach der luetischen Infektion auf. Letztere war nur unzureichend mit Pillen bebandelt worden. Die Affektion war diffus an der linken Brust zu konstatieren in der Größe einer Orange ohne bestimmte Grenzen. Die darüber liegende Haut war mitbeteiligt und sah orangenschalartig aus. Wa.-R. positiv. Auf eine antiluetische Kur trat rasche Besserung ein.

Darier, Ferrand und Mircouche demonstrieren einen Fall von Skleremie oder ödematöser Sklerodermie von Hardy. Die Affektion betrifft fast den ganzen Körper mit Ausnahme der Finger und der Zehen und zeigt sich in Form von skleröser Verdickung der Haut, so daß das Relief der kontrahierten Muskeln verschwindet. Eine Faltenbildung bei seitlichem Druck ist nicht möglich. Keine Pigmentierung, sondern violette Verfärbung der frisch affizierten Partien, während 
die sklerös-atrophischen Hautpartien mehr eine wachsartige Farbe aufweisen. Auf Thyreoideapräparate deutliche Besserung.

Dishussion. Darier möchte die Affektion, gestützt auf den guten Erfolg der Thyrcoideapräparate mit dem Myxödem in Beziehung bringen. Brocq hebt ebenfalls die gute Wirkung der Thyreoidea hervor, lehnt aber, wie für die Dupuitrensche Kontraktur, eine Beziehung zum Myxödem ab. Dubreuilh ist skeptisch in Bezug anf die Wirksamkeit der Thyreoideapräparate bei Sklerodermie und glaubt, daß solche Fälle in einigen Jahren auch spontan heilen können. Milian hebt neben der guten Wirkung der Thyreoidea auch diejenige der Ovarialpräparate bei Frauen hervor. Es handelt sich nach ihm bei Sklerodermie und Sklerodaktylie um eine polyglanduläre Insuffizienz. Ätiologisch komme eventuell auch die Tuberkulose in Frage.

Brocq, Belot und Sankewitch empfehlen zur Behandlung der subkutanen Hautknoten nach Kampferinjektionen die Elektrolyse mit dem negativen Pol. Auch der galvanische Strom und die Röntgentherapie mittels $3 \mathrm{~mm}$ dicken Aluminiumfiltern geben gute Resultate.

$D$ u fou rm en te l. Fall von ausgedehnter $P$ lastik bei einem $K r$ i eg sverletzten. Es wurden dem Manne der Mundboden, die Unterlippe, der Unterkieferknochen und das Kinn ersetzt. Bemerkenswert ist, daß der Mundboden der Wange entnommen wurde und daß sich die äußere Haut der Funktion als Mundboden angepaßt hat. Andererseits wurde die Kinnhant dem Haarboden entnornmen und mittels zwei Stielen der Lappen über das.Gesicht an die benötigte Stelle geklappt. Es bildete sich ein Bart, der vom ästhetischen Standpunkte aus sehr befriedigte. Das Gesamtresultat war ein sebr gutes.

Civatte und Eliascheff demonstrieren einen Fall von Poikilodermie bei einer 4ljährigen Köchin. Betroffen sind die seitlichen Gesichts- und Halspartien, die oberen seitlichen Brustpartien, die Schaltern und die Arme bis zu den Vorderarmen. Die Affektion zeigt sich in Form von netzartig angeordneten braunfleckigen Atrophien z. T. mit kleinen varikösen Gefäßerweiterungen.

Diskussion. Queyrat wänscht Wa.-R., da die Pigmentsyphilis ähnliche Läsionen setzen könne. Nach Darier kommt nach dem histologischen Befunde Syphilis nicht in Betracht.

Balzer und Barthélémy. Fall von fast totaler Alopekie, bei dem post morten eine hochgradige tuberkulöse Entartung beider Nebennieren konstatiert werden konnte.

Dabreuilh demonstriert einen Annamiten aus Indo-China, der an sog. Tokela u leidet. Es finden sich schuppende Plaques im Gesicht, am Hals, den Armen und Händen, am Gesäß, den Knien und den Füßen. Die Herde sind girlandenförmig angeordnet und widerstehen jeglicher Therapie. Die Affektion breitet sich stetig weiter aus. Mikroskopisch lasse sich eine große Menge von Parasitentäden nachweisen.

Dubreuilh demonstriert gewöhnliche und radiologische Photogräphien von einem Leprösen, bei dem es zufolge der Lepra zur Resorption des Metatarsus gekommen ist ohne Sequesterbildung. 
Lered de kritisiert die unzureichende Behandlung und Aufklärung der Syphilitischen in den Spitälern und Polikliniken. Er verlangt vor allem aus Fürsorgestellen nach Quartieren, wo die Luetiker gründlich behandelt werden können. Die großen Spitalkonsultationen hätten mehr eine Sichtung des Materials vorzunehmen und was nicht hospitalisiert wird, den Fürsorgestellen zuzuweisen.

Diskussion. Leredde wird unterstützt von Baudouin, Queyrat und Milian, die ebenfalls die Fürsorgestellen empfehlen, die bemittelten $\mathrm{Pa}$ tienten aber von der Gratisbehandlung ausnebmen möchten. Clément Simon macht auf die Adnexabteilung für Syphilitische in dem Spital von B r o c q aufmerksam, wo für Laboratoriumsuntersuchung, Behandlung usw. genügend gesorgt ist und die eine Fürsorgestelle vollständig ersetzen.

Montpellier und Lacroix. Fall von Mikrosporie in Algier, bedingt durch das Mikrosporon lanosum. Fälle von Mikrosporie, bedingt durch das Mikrosporon Audouini sollen in Algier unbekannt sein. Rasche Heilung mittels Röntgenstrahlen.

Montpellier macht auf die Hänfigkeit der Seborrhoe bei den Muselmanen Algiers aufmerksam und weist andererseits hin auf die Seltenheit der G!atze. Dieses Moment scheint M. nicht so sehr für die seborrhoische Ätiologie der Glatze zu sprechen.

Gougerot, Goulier und Vaslin. Fall von Ulzerationen an der Zunge, die alle Charaktere luetischer Gummata aufwiesen. Die Salvarsanbehandlung gab aber nur einen vorübergehenden Erfolg. Wa.-R. negativ. Mikroskopisch waren fuso-spirilläre Bazillen nachweisbar, so daß die Diagnose auf Angina Vincenti gestellt werden mußte. Lokale Betupfungen mit wässerigen Neosalvarsanlösungen brachten bald völlige Heilung.

Schulmann und Tribaut. Fall von Angina Vincentiam Seitenrand der Zunge, der unter der Diagnose Primäraffekt ins Spital geliefert wurde. Mikroskopisch handelte es sich um die Symbiose von Spirillen mit fusiformen Bazillen. Klinisch um eine übelriechende Ulzeration, die in der Mitte einen schmutzigen Belag aufwies mit Drüsenschwellungen am Unterkiefer. Auf Betupfungen mit Methylenblau und Spülungen mit $\mathrm{H}_{8} \mathrm{O}_{2}$ trat in 14 Tagen Heilung ein. Die Wa.-R. war vor und nach der Behandlung negativ.

Diskussion. Goubeau will zablreiche Fälle von Angina Vincenti beobachtet haben. Pulverrilh betont, daß diese Ulzerationen nicht selten bei Stomatitis ulcerosa vorkommen und therapeutisch gut durch Betupfung mit Chromsäure zu beeinflussen seien. Queyrat betont die gute Wirkung der lokalen Novarsenobenzolbetupfungen, während das Medikament intravenös zu versagen scheine.

Lévy-Franckel berichtet über eine „crisenitritoide“ nach einer intravenösen Injektion von kolloidalem SchwefelQuecksilber. Es handelt sich um eine 20jährige Patientin, die wegen Iues zunächst mit $\mathrm{Hg}$ und einigen Novarsenobenzol- und Disodo-LuargolInjektionen behandelt worden war. Nach den letzteren trat jeweilen kurz dauerndes Fieber und etwas Unwohlsein auf. Nach einer intravenösen Injektion von 0.01 kolloidalem Schwefel-Quecksilber traten sofort fol- 
gende Symptome auf: Schnupfen mit starker seröser Sekretion, Behinderung der Nasenatmung mit Dyspnoe, Kongestion des Gesichtes, Heiserkeit, Schwierigkeiten im Schlucken, Zeichen von akuter Bronchitis. Subjektiv Geschmack von $\mathbf{S}$ im Munde. Während der Nacht Schüttelfrost und starkes Kältegefühl der Extremitäten. Am folgenden Tage waren sämtliche Erscheinnngen verschwunden.

Bouveyron empfiehlt zur Behandlung der geschlos senen Gonorrhoe - Epididymitis, Arthritis gonorrhoica, Salpingitis usw. intramuskuläre Enesolinjektionen. Sie sullen ähnlich wirken wie die Gonokokkenvakzine.

Gouge rot demonstriert einen Soldaten mit einer Narbe nach einer Kriegsverletzung an der Hand. Die Narbe hat die Eigentümlichkeit, $\mathrm{daB}$ sie verrukös und lichenifiziert ist und stark juokt. Die Begrenzung ist scharf und der Saum hat einen rotvioletten Farbenton.

Gougerot, Clara und Boanin demonstrieren einen Fall von pigmentierter Papillomatose mit Porokeratose an den Handtellern und papulovesikulōsen Effloreszenzen. Sie stellen keine bestimmte Diagnose.

\section{Sitzung vom 19. Juli 1919.}

Der neugewählte Vorsitzende Brocq hält eine bemerkenswerte Rede, worin er namentlich die jüngere Generation zu energischer Arbeit auffordert. Er wünscht eine Organisation der Arbeit und Zusammen. schluß der wirksamen Kräfte, um möglichst fruchtbringende Resultate zu bekommen. Frankreich dürfe nicht nur nach dem Ausland Umschau halten, sondern müsse die großen Probleme der Dermatologie selbst lösen. Dazu sei es kraft seines kritischen Geistes berufen.

Pinard demonstriert einen Fall von syphilitischer Super. infektion. Im November 1918 Primäraffekt mit positiver Wa.-R. Pat. bekommt 2.3 $g$ Novarsenobenzol. Klinisch geheilt, serologisch nicht mehr untersucht. Am 14. Mai 1919 verkehrte Pat. mit seiner Frau, die an abheilenden sekundär luetischen Erscheinungen litt. Ein Monat später wieder Ulzus an der Penishaut vom Charakter eines Primäraffektes mit Drüsenschwellungen in den Leisten und kurze Zeit darauf zeigten sich sekundär-luetische Effloreszenzen am Rumpf. Wa.-R. positiv. P inard glaubt, daB es sich um eine Superinfektion handle in Anbetracht namentlich des kurzen II. Inkubationsstadiums - 14 Tage - zwischen primärer und sekundärer Erscheinung.

Périn demonstriert einen Fall von Acanthosis nigricans bei einer 46jährigen Frau, wobei die Veränderungen der Mundschleimhaut bemerkenswert sind. Die $Z$ unge ist in ihrem vorderen Bereiche zottig, ebenso die Oberlippe, während die Unterlippe einen mehr samtartigen Charakter aufweist. Am harten Gaumen finden sich hufeisenförmig angeordnete papillomatöse Wucherungen von 2-3 mm Größe. Auch an der Wangenschleimhaut und am Zahnfleischrand finden sich leicht papilläre Wucherangen. Das histologische Bild ist typisch. Von 
karzinomatösen Veränderungen innerer Organe kann bis jetzt nichts nachgewiesen werden trotz Abmagerung und allgemeiner Asthenie.

Diskussion. Brocq macht auf die scharfe Begrenzung der Läsionen am harten Gaumen aufmerksam, was bewirke, daß die Veränderungen mehr einer Papillomatose gleichen.

Thibierge und Gastinel demonstrieren eine bandförmige Sklerodermi e, die von dem äußeren Ende der rechten Augenbraue über die Stirn bis zur Gegend der großen Fontanelle verläuft. Im Bereiche der Parietalgegend zeigt sie eine leichte Vertiefung und in diesem Bereiche ist auch der Knochen leicht atrophisch.

Tzanck. Fall von Encephalitis acuta bei einer 27jährigen Frau, die einige Jahre zuvor Dosen von 0.9 Novarsenobenzol gut vertragen, auf Dosen von 0.6 aber auch schon Kopfschmerzen bekommen hatte. Dazu entzündliche Erscheinungen von Seite der Zerebrospinalflüssigkeit - Albuminurie, Lymphozytose, vermehrter Druck. Wa.-R. blieb lange Zeit positiv. Pat. bekam dann 4 Jahre lang nur Hg. Es wurde dann wieder ein Versuch mit Novarsenobenzol gemacht. Auf 0.075 Übelkeit. Anf $0 \cdot 1$, die 8 Tage später injiziert wurden, sofort Kongestion und Ameisenlaufen im Gesicht, starke Kopfschmerzen, nachber Gesichtsblässe, verlangsamte Atmung, Herztöne kaum hörbar. Puls nicht mehr fühlbar. Sphinkterenlähmung. Bewußtseinsverlust. Koma dauert 20 Minuten. Nach 10 Minuten langer künstlicher Atmung kommt das Bewußtsein wieder. Aber ungefähr 6 Stunden lang klagt Pat. noch über Übelkeit, Kopfschmerzen, erschwerte Respiration, Erbrechen und Diarrhoe. Sie bleibt sehr blaß. Therapeutisch bekam Pat. Adrenalin intern, intramuskulär und subkutan, je $1 / 2 m g$, später $1 / 4 m g$ intravenös, worauf rasche Besserung und allmählich komplette Heilung eintrat.

Lacapère und Galliot demonstrieren einen Fall von syṕhilitischer Reinfektion. Pat. soll vor 10 Jahren schon einen Primäraffekt gehabt haben, der auf eine einzige intramuskuläre 606 Injektion geheilt sei. Nachher keine Behandlung mehr bis zur gegenwärtigen Infektion, die sich in Form eines sekundären luetischen Exanthems, mit Resten eines Primäraffektes und positiver Wa.-R. zeigt. Die erste Beobachtung scheint allerdings nicht ganz beweisend gewesen zu sein.

Carle macht auf die Wichtigkeit der klinischen Untersuchung bei der Interpretation der Wa.-R. aufmerksam, sowie auf die exakte Technik der Ausführung der Reaktion. Nur dann habe die Wa.-R. einen unbestreitbaren Wert. Dieses Urteil fällt Carle gestützt auf seine Erfahrungen in der Armee. In einem gut geleiteten Laboratorium hatte er folgende Ergebnisse: Im I. Stadium d. h. 50 Tage post infectionem hatte er $80 \%$ positive Reaktionen, im II. Stadium $93 \%$ und im III. Stadium $86 \%$. Bei nicht Spezifischen bekam er $90 \%$ negative Resultate.

Goubeau empfiehlt folgende Technik der Wa.-R., um Fehlerquellen möglichst zu eliminieren: 1. Es muß stets die Originalmethode nach B ordet-Wassermann gemacht werden mit auf $56^{0}$ erhitztem Sermm und mit einem Antigen, das aus hereditär-syphilitischer Leber hergestellt und nach Konvention vereinheitlicht ist; 2. dieselbe Methode muB mit 
frischem Serum nach Hallion-Bauer gemacht werden; 3. die Ersatzmethoden dürfen nie allein gemacht werden; 4. es muß eine einheitliche MeBmethode eingeführt werden. Goubea u gibt der Skala von Vernes den Vorzug. Leredde erklärt, gestützt auf seine Erfahrungen, daß es nicht genüge, einen frisch infizierten Luetiker Wassermann negativ $z u$ bringen, um eine Heilung zu garantieren. Stets müsse noch die Lumbalflüssigkeit untersucht werden. Es zeigen sich da häufig noch entzündliche Erscheinnngen auch bei negativem Blutbefund. Gut sei es, wenn die Lues auch noch leicht behandelt werde, wenn die Lumbalflüssigkeit normal geworden sei. Er erwähnt einen Fall von Roseola, der von Anfang an sehr energisch mit Novarsenobenzol behandelt wurde und bei dem auch die Lumbalflüssigkeit nach der Behandlung entzündliche Erscheinungen aufwies. Die Wa.-R. war trotz wiederholter Behandlung nie ganz negativ zu bringen. Es zeigen sich weitere Erscheinungen in Form von Zittern der Hände, Steigerung der Patellarreflexe und Abnahme des Gedächtnisses. Le redde vermutet, dafs es sich in diesem Falle um eine beginnende Paralyse haudeln könnte.

Pinard beobachtete 2 Fälle von Pityriasis rosea im 1. Inkubationsstadium der Lues. Beim 1. Fall bestand die Eigentümlichkeit, daß die Eruption etwa 6 Monate dauerte und mit braunen Pigmentflecken abheilte. Pinard will die Affektion öfters bei Individuen gesehen haben, die allgemein leicht mitgenommen waren und besonders ist ihm aufgefallen, daß beginnende Tuberkulöse an Pit. rosea erkrankten. Es müsse gewissermaßen ein Terrain vorhanden sein, damit sich die Krankheit entwickeln könne. Pit. rosea-Kranke müssen auch späterhin noch einige Zeit unter Kontrolle gehalten werden.

Diskussion. Clément Simon bat in den letzten 2 Monaten etwa 10 Fälle von Pityrosea beobachtet, die aus der gleichen Gegend - Umgebung von !aris - stammten. Die Affektion scheine zu gewissen Zeiten einen epidemischen Charakter zu haben. Brocq will die Pityriasis rosea in dem Entwicklungsstadium der Lues häufig beobachtet haben. Auch bei Allgemeinstörungen komme sie vor, aher aush bei sonst gesunden Leuten.

Pinard. 2 Fälle von zirzinären Tuberkuliden am Gesäß, die die Form eines $\mathrm{C}$ hatten. Wa.-R. bei beiden Fällen negativ, die antiluetische Kur versagte. Klinisch glichen die Fälle einer tubero-serpiginösen III. Lues.

Pinard berichtet über 4 Fälle von Paraplegie nach Arsenbehandlung der Lues. Der 4. Fall heilte nach Wiederaufnahme einer sehr energischen Arsenbehandlung, so daß $P$ inard eine Arsenintoxikation für ansgeschlossen hält und eher geneigt ist, eine Herxheimersche Reaktion anzunehmen, weshalb er eine weitere As-Behandlung für indiziert hält.

Diskussion. Leredde scheint der letzte Fall Pin ards zu beweisen, daß es sich in solchen Fällen um eine Myelitis syphilitica handle.

Gougerot hat 3 Fälle von angiomatösen $N$ ävi beobachtet, die sich bei einem Fall nach einer Verletzung und in den zweí anderen Fällen nach Erfrierung entwickelt hatten.

Gougerot demonstriert einen Fall von ausgedehnter Vitiligo, der sich in der Umgebung einer Kriegsverletzung entwickelt hatte und nach der Operstion zum Stillstand kam, d. h. die Vitiligo entwickelte 
sich nicht mehr weiter. Nichts vou Nervenstörungen oder luetischen Erscheinungen nachweisbar. G. hält den Vitiligo für eine näviforme Dystrophie, bei der die hereditäre tues eine der Hauptursachen darstelle. Die Syphilis komme aber dabei nicht einzig in Frage.

\section{Sitzung vom 13. November 1919.}

Darier teilt ergänzend zu seinem in der Sitzung vom 10. Juli vorgestellten Falle von $\mathrm{Akanthosis} \mathrm{nigricans} \mathrm{mit,} \mathrm{daß} \mathrm{Pat.} \mathrm{nun}$ deutliche Zeichen eines abdominalen Tumors darbiete und zwar handle es sich wahrscheinlich um ein Magenkarzinom mit Lebermetastasen.

Goubeau demonstriert eine Arbeit von Lagage, der einige technische Angaben macht, um die Unregelmäßigkeiten im Ausfall der Wa.-R. nach Möglichkeit zu vermeiden. Er verlangt namentlich ein praktisch einheitliches Meerschweinchenserum.

Fernet und Nahan. Fall von Kalkablagerungenim Unter hautzellgewebe der Finger bei einem 16̣jährigen Mädchen. Die Größe der Knoten schwankt von Stecknadelkopf- bis Haselnußgröße. Ee handelt sich um Ablagerungen von phosphorsaurem und koblensaurem Kalk. Es besteht eine leichte Akroasphyxie der Finger und hin und wieder das Phänomen der sog. toten Finger. Um die Kalkablagerung findet sich eine Reaktion des Bindegewebes in Form von großen Riesenzellen mit sehr zahlreichen Kernen. Die Autoren vermuten, es könnte sich um die von Milia n beschriebenen "Granulomes calcaires souscutanés“ handeln.

Diskussion.' Milian bestätigt die Analogie dieses Falles mit seinen publizierten Fällen. Auch Larier schließt sich dieser Auffassung an.

Milian, Sauphar und Thibaut demonstrieren einen Fall von Granuloma annulare bei einem 8jährigen Kinde. Die Wa.-R. war einmal positiv, nachher ohne spezifische Behandlung negativ. Neosalvarsaninjektionen blieben ohne Einfluß auf die Affektion. Die Wa.-R. blieb ebenfalls negativ. Histologisch handelt es sich um eine Hyper- und an der am stärksten vorspringenden Stelle um eine Parakeratose. In der Kutis Degeneration des Bindegewebes und Anhäufung von epitheloiden Zellen und Riesenzellen ohne Verkäsung. Die Autoren glauben, daß dieser Befund zu Gunsten einer tuberkulösen $\ddot{A}$ tiologie des Gr. annulare sprechen.

Diskussion. Darier empfiehlt therapeutisch Skarifikationen, da man oft nach Probeexzisionen Teile des Tumors verschwinden sehe. Milian sah nach der Biopsie bei diesem Falle keine Besserung. Er will bei einem anderen ausgedehnten Falle Übergänge von granulomaähnlichen Knoten zu lupösen Knötchen gesehen haben.

Milian und Blum demonstrieren einen Fall von Erythem mit Purpura bei einem 4:jährigen Manne, der Zeichen von Plethora und zugleich von leichter Anämie darbot. Es handelte sich um größere Plaques an den Armen und Beinen, am Abdomen und z. T. am Rücken zwischen den Schulterblättern. Die Plaques bestehen aus roten Flecken, untermischt mit Purpuraflecken. Auf Druck vehmen die Erythemflecke 
einen gelblichen Farbenton an. Allgemeinbefinden sonst gut. Kein Jucken. Die Affektion besteht seit Wochen und ist schon zum 3. Male aufgetreten. Ursache nicht eruierbar.

Diskussion. Goubeau will einen gleichen Fall beobachtet haben bei einer Patientin, die anno 1916 an Skorbut litt. Darier meint, man müsse bei solchen Fällen auch an die Poikilodermia Jakobi denken.

Clément Simon wünscht, daß ron der Gesellschaft die Frage des Ehekonsenses der Syphilitiker neu studiert werde.

Milian empfieblt statt der alten Behandlungsmethode der Skabies mittels Bürste und Schwefelsalbe die zweimalige Anwendung der Kaliumpolysulfatsalbe und nachheriges Bad. Die Methode sei billiger, erübrige das Bürsten mit Schmierseife, das schmerzhaft und oft irritierend sei. Zngleich schone sie die Kleider und setze weniger Infektionen.

Diskussion. Die Polysulfatsalbe wird weiter empfohlen von Darier und Goubeau. Sabouraud empfiehlt, die alte und neue Methode nebeneinander zu prüfen, was von Thibierge angenommen wird.

Bory, L., tritt für die parasitäre Natur der Psoriasis ein und berichtet über einen Fall, bei dem sich im Anschlusse an Wanzenstiche eine Psoriasis entwickelt hatte und zwar wandelten sich die Stiche nach 8 Tagen allmäblich in typische Psoriasisherde um. Allmählich breitete sich die Affektion auch über nicht gestochene Stellen aus, um schlieblich den ganzen Körper zu überziehen. Mikroskopisch fand Bory im Reizserum kleine unregelmäbige Stäbchen, die sich nach Tanninbeizung mittels polychromem Methylenblan gefärbt batten. Die Stäbchen sollen am ehesten dem Mikrosporon minutissimum geglichen haben. Kultur nicht möglich. In vielen anderen Fällen fand der Autor die Stäbchen nicht, so daß er an Verunreinigungen denkt. Dor Pat. betrifft einen Krankenwärter, der Psoriasiskranke zu behandeln hatte nnd B. glaubt, daß die Möglichkeit einer Übertragung des Psoriasisvirus mittels Wanzen möglich gewesen sei.

Diskussion. Darier wünscht, daß die Frage der Psoriasis einer speziellen Kommission zum Studium übertragen werde.

Leredde berichtet über einen Fall von papulo-vesikulösem Ekzem sowie über einen Fall von Prurigo diathésique, die durch Novarsenobenzolinjektionen gebessert wurden. Beim ersten Fall handelte es sich um eine luetische Hypertrophie der Leber und das Ekzem trat erst nach der 2. Injektion auf, versehwand aber dann auf weitere Injektionen total, ebenso der Juckreiz. Der 2. Fall betraf ein 13jähriges Fräulein, dessen Vater an Pupillendifferenz leidet, die von Leredde auf Lues zarückgeführt wird. Bei der Pat. bestanden lichennifizierte Plaques am Hals und den Gelenkbeugen der Arme und Beine, die wir als neurodermitisch zu bezeichnen pflegen. Dazu litt sie an astbmatischen Anfällen. Auf Novarsenobenzol zunehmende Besserung der Plaques und Verschwinden des Asthmas.

Bei einem 3. Falle handelte es sich um ein subakutes Ekzem an den Wangen bei einem Mädchen, das von syphilitischen Eltern 
stammte, ohne dab es Zeichen von hereditärer Lues darbot. Auf Hektininjektionen Heilung.

Auch einen Fall von generalisiertem Pruritus mit diffuser Pigmentierung bei negativer. Wa.-R. sah L. nach Hektininjektionen verschwinden. Der Mann der Patientin litt an Lues.

Leredde glaubt, es könnte sich in diesen Fällen um sogenannte Hämatodermitiden luetischen oder paraluetischen Ursprungs handeln.

Diskussion. Renault macht darauf aufmerksam, daß man schon lange die günstige Wirkung des Arsens auf die Prurigo Hebrae kenne, ohne daß deshalb dje luetische Natur der Affektion involviert sei. Milian sagt, daß Fournier den bereditär-luetischen Ursprung der Prurigo Hebrae öfters nachgewiesen habe.

Balzer demonstriert alte Ampullen von Novarsenobenzol und Galyl, die in Glykose oder $\ddot{l} l$ gelöst waren und die zur intramuskulären Injektion dienten. Die Ampullen wiesen eine veränderte Farbe auf: statt Bernsteingelb waren sie rot bis bräunlich. B a izer mußte vorsichtsbalber hin und wieder solche Ampullen vom Gebrauche ausschließen, sah aber nie irgendwelche Intoxikationserscheinungen dabei. Ötters sollen die dunkleren Ampullen sogar etwas weniger Schmerzen verursacht haben, als die normalfarbigen.

Liskussion. Moun yrat will diese Veränderungen nicht auf das Präparat selbst, sonclern aut die Vehikel, speziell die Glykose, zurücktübren, die manchmal nicht rein sei und öfters Spuren von Eisen enthalte. Die Veränderung soll nicht eintreten, wenn stat Glykose Iävulose verweudet werde. Nach Balzer ist die Farbenänderung auch bei alten Ölampullen zu konstatieren.

Burnier. Fall von Primäraffekt am Vorderarm nach einer Brandwunde. Der Fall betrifft einen Heizer, der neben einem Lokomotivführer diente, der an Plaques muqueuses litt. B urnier vermutet Übertragung des luetischen Virus durch den letzteren mittels Lappen oder anderer Gebrauchsgegenstände.

Burnier. Fall von $S k$ lerodermie, die nach einem Trauma an den Unterschenkeln an der Stelle des letzteren autgetreten ist. Auf die ödematöse folgte die skleröse Phase.

Montpellier und Laurens. Fall von Tabes bei einem eingeborenen Muselmanen Nordafrikas. Die Symptome sind typisch. Luetische Infektion anno 1911 zugegeben. Daneben war Patient früher starker Alkoholiker. Wie weit der Alkoholismus am Ausbruch der Tabes beteiligt ist, wagt der Autor nicht zu entscheiden.

Uruena, J. G. Fall von Xeroderma pigmentosum bei einem. 8 Jahre alten Mexikaner. Die Affektion hatte die Eigentümlichkeit, tast über den ganzen Körper auggebreitet zu sein mit Veränderungen an der Zungenspitze, wo kleine rote und achromische Flecke bestanden. Die Affektion soll im Anschlusse an Masern im Alter von $1 \frac{1}{2}$ Jahren begonnen haben.

Sitzung vom 18. Dezember 1919.

Spillmann und Watrin. Fall von Xanthoma papulosum et generalisatum bei einem 9jährigen Knaben, der mit Cholesterinä- 
mie und Diabetes insipidus verbunden war. In den histologischen Präparaten sollen hauptsächlich Cholesterinäther, weniger neutrales Fett in den Zellen nachweisbar gewesen sein.

Gastinel und Legrain demonstrieren einen 45jährigen Schubmacher, der an einem Primäraffekt des harten Gaumens, übergreifend auf das linke Zahnfleisch, mit sekundär-luetischen Erscheinungen leidet. Zugleich besteht eine Erosion von 20 Centimesstückgröße am vorderen Zungenrand rechts. Pat. ist zahnlos und trägt eine Prothese. Bei der Arbeit nimmt er die Prothese heraus und hat die Gewohnheit, die kleinen Nägel in den Mund zu nehmen, die bei der Arbeit verwendet werden. Da diese Nägel häufig der Schachtel eines Nachbargesellen entnommen werden, vermuten die Autoren, es könnte auf diesem Wege eine luetische Übertragung stattgetunden haben. Bestimmte Anhaltspunkte hiefür haben sie aber nicht. Eine venerische Infektion lehnen sie $a b$, da Patient seit 10 Monaten keinen Verkehr gehabt haben soll.

Legrain und Pellier demonstrieren einen Fall von Sporotrichose mit einer krustösen, verrukösen Läsion an der Nase und ektymatoiden Läsionen der Extremitäten ohne Lymphangitis. Kulturell kunnte das Sporotrichon nachgewiesen werden.

Diskussion. Gougerot fragt nach der Behandlung. Nach ihm ist die verruköse Form viel s'hwerer zu heilen als die ulzeröse, eine Ansicht, die auch von Brocq geteilt wird. Gou gerot hat in solchen Fälen lokale Injektionen mit Jodjodkalilösungen gemacht. In einzelnen Fällen hat er zur Radiotherapie und Heißluttapplikation gegriffen. Simon hat bei einem Falle von wuchernder a ktinomykose mit gutem Erfolge intravenöse KJ-Injektionen in Dosen von 0.5 jeden 2. Tag gemacht.

Parin und Périn demonstrieren einen Fall von Lupus erythematodes, bei dem neben der typischen Schmetterlingsform im Gesicht kleinere Herde im Gesicht, an der Oberlippe und an den Iländen bestehen. Daneben lassen sich typische große Plaques von Lupus erythematodes an beiden FuBsohlen konstatieren.

Diskussion. Leredde fragt nach der Wa.-R. Nach Parin wurde sie nicht ausgeführt. Brocq bezeichnet den Lupus erythematodes der Plantae als eine große Seltenheit.

Montpellier und Marchand haben innerhalb von 2 Jahren 19 Fälle von malignen Haut $t$ umoren bei den Eingeborenen Nordafrikas beobachtet, von denen 18 Epitheliome und nur ein Fall ein Sarkom war. Sie haben auch die übrigen Ärzte, die hauptsächlich Eingeborene zu behandeln haben, nach der Häufigkeit der malignen Haùt. tumoren gefragt und mit ihren Beobachtungen übereinstimmende Antworten bekommen. Es wurde allgemein beobachtet, daß die malignen Tumoren bei den Eingeborenen relativ häufig vorkommen und daß der Krrebs bei weitem das Sarkom überwiegt.

$\mathrm{L}$ a c a père hatte auf einer längeren sanitarischen Inspektion in Marokko Gelegenheit, den Ursachen der Malignität der Lues bei den eingeborenen Arabern nachzuforscben. Er kommt zum Schlusse, daB es hauptsächlich die Malaria ist, die den Blutdrack stark erniedrigt und 
dadurch Anlaß zur Malignität der Lues gibt. Durch den niedrigen Blutdruck komme es in den luetischen Läsionen weniger zur Resorption, sondern zur Nekrose und zum Zerfall. Auch der Mangel an Alkohol führe zu einem niedrigen Blutdruck. Nach La cap ère erklärt sich daraus das häufige Vorkommen der Malignität bei den Arabern, die Abstinenten sind und auch sehr häufig an Malaria leiden, während die Senegalesen, die mehr auf den Kämmen des Atlas wohnen, von Malaria trei bleiben und zugleich Wein trinken, sehr selten an maligner Lues erkranken. Ein spezielles Virus brauche demgemäß nicht angenommen zu werden. Das erkläre auch das Versagen der Hg-Therapie bei maligner Lues, da $\mathrm{Hg}$ den Blutdruck herabsetze, während die Arsenpräparate mit der blutdrucksteigernden Wirkung ein rasches Verschwinden der ulzerösen Läsionen bewirke.

Diskussion. Queyrat sagt, er hätte schon lange den arteriellen Blutdruck bei Lues I und II studiert und getunden, daB er dabei stets herab. gesetzt sei. Man müsse zwischen schwerer und frühzeitiger maligner Syphilis unterscheiden. Bei der ersteren finde man Spirochäten, bei der letzteren sollen sie fehlen. B roc $q$ hat trüher die maligne Lues mit JodHg-Kakodylat behandelt.

Lévy-Franckel und Jacob ließen bei 49 Psoriatikern die Lungen radiologisch untersuchen und es wurde in 16 Fällen eine normale und $27 \mathrm{mal}$ eine pathologisohe Lunge festgestellt, während in 6 weiteren Fällen die Läsionen äußerst minimal waren. Bei den stärkeren Veränderungen fanden sich Marmorierungen, Zwerchfellsadhärenzen, graue Lungen mit Spitzen, die sich beim Husten nicht authellten, skleröse Perıbronchitis. Die Autoren wollen aus diesen Beobachtungen den SchluB ziehen, daß ein tuberkulöses Terrain der Entwicklung einer Tuberkulose günstig sei.

Eliascheff demonstriert einen Fall von Epilepsie mit Hautveränderungen, die sich hauptsächlich auf die Knie und Ellbogen, Oberschenkel, Gesäß, Rücken, Gesicht und Ohren beschränken. Die Läsionen bestehen in hanfkorn- bis 2 frankstückgroßen und größeren braunen Flecken und flachen Knoten. Stellenweise fließen die Knoten zu polyzyklisch geformten Infiltraten zusammen. Von den größeren Flecken zeigen einige einen erhabenen Rand und ein etwas eingesunkenes Zentrum ohne Atrophie. Keine Transparenz, keine Ulzeration, keine Desquamation, weder Jucken noch Schmerzen. Die Läsionen sollen sich langsam seit 1913 entwickelt haben. Seit jener Zeit besteht auch die Epilepsie. Histologisch handelt es sich um ein entzündliches Infiltrat, das vorwiegend aus jungen Bindegewebszellen, Polynukleären und Eosinophilen besteht. Stellenweise sind auch epithelioide und Pigmentzellen konstatierbar. Das elastische Gewebe ist verschwunden. Nichts von Xanthom oder Lepra. Es handelt sich nach dem Vortragenden um eine infektiöse Neubildung, die gewisse Analogien mit dem hämorrhagischen Pigmentsarkom Kaposis aufweist. Eine bestimmte Diagnose kann er nicht stellen.

Diskussion. Brocq hält die Diagnose für außerordentlich schwierig und glaubt am ebesten an eine Sarkomatose, die noch nicht beschrieben 
sei. Darier, aus dessen Abteilung der Fall stammt, glaubte zuerst eine Lepra diagnostizieren zu müssen, nachher ein Xanthom, bis ihn die histologische Untersuchung diese Diagnosen fallen ließ̧. Er hält die Krankheit für ein infektiöses Neoplasma, das große Ähnlichkeit mit einem früher beobachteten Fall aufweist. Er glaubt eine pigmentierte Form des Sarkoms Kaposi annehmen zu müssen oder etwas, was diesem sehr nahe stebt.

Simon, C., demonstriert einen Mann, bei dem innerbalb 6 Monaten $2 \mathrm{Schankerrezidive} \mathrm{auftraten.} \mathrm{Der} \mathrm{erste} \mathrm{Primäraffekt} \mathrm{wurde}$ am 24. Juni 1919 konstatiert bei negativer Wa.-R. Pat. bekam $4 g$ Novarsenobenzol. Normale Heilung. 3 Wochen nach der letzten Injektion wíeder Schanker an derselben Stelle. Hecht negativ. Keine sekundären Erscheinungen. Bekommt $5 g$ Novarsenobenzol. 3 Wochen nach der letzten Injektion wieder Rezidiv in situ. Verkehr, aber nur mit Kondom, zugegeben. Es handelt sich nach Simon in diesem Falle weder um Reinfektionen noch sekundäre Erscheinungen, sondern um lokale Rezidive durch nicht völlig vernichtete Spirochätenreste.

$\mathrm{H} \mathrm{udelo}$ und $\mathrm{M}$ on $\mathrm{t} \mathrm{I}$ a $\mathrm{r}$ demonstrieren einen Fall von $\mathrm{Pe} \mathrm{m}$ phigus mit Atrophie der Handrücken und Zystenbildung. Der Fall wurde schon im Alter von 10 Jahren in der Sitzung vom 6. November 1913 in der Gesellschaft vorgestellt. Auffallend war die Kombination mit Hypertrichosis im Gesicht und an der Streckseite der Arme, einer star* ken Pigmentierung des Gesichtes und Halses, sowie einer gewissen Adipositas des Bauches. Urin stets dunkel durch ein eigentümliches Pigment ohne Hämoglobin. Der Vater der Patientin litt an Lues. Das Kind zeigte eine MiBbildung der Zähne und eine suspekte Wa.-R. Novarsenobenzol und Hochfrequenzströme ergaben keine Besserung. Vom Oktober 1917 an bekam das Mädchen 15 Tage Ovarialextrakt, 15 Tage Pause, 15 Tage Nebennierenextrakt, dann wieder ebenso lange Pause, in gleicher Weise Hypophysen- und Thyreoideaextrakte mit den respektiven Pausen. Erfolg sehr gut. Die Blasenbildung verschwand allmählich, die großen Gesichtshaare verschwanden und machten Flaumhaaren Platz, die Pigmentierung ging zurück. Pat. wurde menstruiert. Nach einem Jahr Rezidiv. Sämtliche Erscbeinungen wurden rückfällig, schlimmer als zuvor. Auf die frühere Behandlung sofort wieder Erfolg. Die Autoren nehmen an, daß es sich in diesem Falle um eine vielleicht durch das syphilitische Virus erzeugte Dystrophie der endokrinen Drüsen handle, die nur durch die Polyopotherapie beeinflußt werden könne.

Diskussion. Gougerot und Brocq wollen wissen, welche von den endokrinen Drüsen die Haare zum Ausfall bringe. Abadie wünscht, daß man es nicht mit einer Polyopotherapie versuche, sondern daß man an Hand dieses Falles die Wirkung jedes einzelnen Extraktes prüfen soll. Simon glaubt, man müsse in solchen Fällen die Behandlung fast dauernd fortsetzen, am das gute Resultat beibehalten zu können. Brocq wünscht, daß untersucht werde, ob eines oder mehrere Extrakte in diesem Falle wirksam seien. Max Winkler (Luzern). 